\title{
Fatores de risco para mortalidade neonatal e pós-neonatal na Região Centro-Oeste do Brasil: linkage entre bancos de dados de nascidos vivos e óbitos infantis
}

\author{
Risk factors for neonatal and post-neonatal \\ mortality in the Central-West region of Brazil: \\ linked use of life birth and infant death records
}

Otaliba Libânio de Morais Neto 1

Marilisa Berti de Azevedo Barros 2

\footnotetext{
1 Departamento de Medicina Tropical, Saúde Coletiva e Dermatologia, Instituto de Patologia Tropical e Saúde Pública, Universidade Federal de Goiás. Rua Delenda Rezende de Melo s/no, Setor Universitário, Goiânia, GO 74605-050, Brasil. olmorais@zaz.com.br 2 Departamento de Medicina Preventiva e Social, Faculdade de Ciências Médicas, Universidade Estadual de Campinas. C. P. 6111, Campinas, SP 13083-970, Brasil. abarros@correionet.com.br
}

\begin{abstract}
This article focused on risk factors for neonatal and post-neonatal mortality by linking live births and infant death records. The study was conducted in the municipality of Goia nia, in the Central-West region of Brazil. A total of 20,981 live births and 342 infant deaths constitute the retrospective cohort. Neonatal and post-neonatal mortality risks were estimated in this cohort study of live births by logistic regression. In the neonatal period, the highest ORs were for delivery in public hospitals (OR $=2.28$; 95\% CI 1.57-3.32), pre-term neonates $(O R=8.94 ; 95 \%$ CI 5.85-13.67), and low birth weight (OR = 8.92; 95\% CI 5.77-13.79). Cesarean delivery appeared as a protective factor $(\mathrm{OR}=0.58 ; 95 \% \mathrm{CI} 0.43-0.78)$. For post-neonatal mortality, the highest ORs were for illiterate mothers $(O R=6.25 ; 95 \%$ CI 1.25-31.27), low birth weight $(O R=3.12 ; 95 \%$ CI 1.67-5.84), and delivery in public hospitals (OR =2.65; 95\% CI 1.13-6.23). The linkage identified socioeconomic variables that were more important risk factors for post-neonatal than neonatal mortality.
\end{abstract}

Key words Infant Mortality; Neonatal Mortality; Risk

Resumo O objetivo do presente artigo foi investigar fatores de risco para mortalidade neonatal e pós-neonatal valendo-se do procedimento de linkage de bancos de dados. A coorte foi constituída de 20.981 nascidos vivos em Goiânia, GO, entre os quais ocorreram 342 óbitos. Fatores de risco foram estimados utilizando regressão logística. Para o período neonatal, foram encontradas odds ratio (OR) significativas para nascidos em hospital público-estatal (OR = 2,28; IC 95\% 1,57-3,32), prematuro $(O R=8,94$; IC 95\% 5,85-13,67), baixo peso ao nascer $(O R=8,92 ;$ IC 95\% 5,77-13,79) $e$, como fator de proteção, o nascimento por cesariana $(O R=0,58 ;$ IC 95\% 0,43-0,78). Os subgrupos expostos a um maior risco de mortalidade pós-neonatal foram: nascidos vivos de mãe sem instrução $(O R=6,25$; IC 95\% 1,25-31,27), baixo peso ao nascer $(O R=3,12$; IC 95\% 1,67-5,84) e nascimento em hospital público-estatal $(O R=2,65$; IC 95\% 1,13-6,23). Os resultados mostram a viabilidade do procedimento para a identificação de fatores de risco para mortalidade infantil e reafirmam a importância dos fatores sócio-econômicos como fatores mais relacionados ao componente pós-neonatal da mortalidade infantil.

Palavras-chave Mortalidade Infantil; Mortalidade Neonatal; Risco 


\section{Introdução}

No Brasil, observou-se um decréscimo de $71 \%$ na taxa de mortalidade infantil entre os anos de 1930 e 1990, embora tenham sido observados períodos de estabilidade e até mesmo de elevação desses coeficientes. As maiores reduções da mortalidade infantil ocorreram nas Regiões Sul, Sudeste e Centro-Oeste (Simões \& Monteiro, 1995). Mesmo com importante queda nas taxas de mortalidade infantil, persistem diferentes níveis e padrões de declínio das taxas entre regiões geográficas e entre subgrupos populacionais no interior das regiões, estados e municípios (Paim \& Costa, 1993; Monteiro \& Nazário, 1995; Simões \& Monteiro, 1995).

O componente pós-neonatal é o responsável pela maior parte da redução da mortalidade infantil nas últimas décadas, ao passo que o componente neonatal representa a maior parcela da taxa de mortalidade infantil, principalmente nas regiões onde as taxas são menores (Maranhão et al., 1999).

O estudo de fatores de risco para mortalidade infantil neonatal e pós-neonatal, compreendidos como indicadores das várias dimensões das condições de vida, é uma estratégia útil, por várias razões: 1) para compreender alguns elementos da cadeia de eventos relacionados à determinação da mortalidade infantil; 2) para identificar grupos expostos a diferentes fatores de risco; 3) para detectar necessidades de saúde em diferentes subgrupos populacionais, com objetivo de subsidiar intervenções voltadas para a redução do risco de morte nos períodos neonatal e pós-neonatal (César, 1990; Battellino \& Bennum, 1991; Castellanos, 1991).

Vários estudos investigaram fatores de risco para mortalidade infantil utilizando o procedimento de linkage de bancos de dados de óbitos e nascimentos (Semenciw et al., 1986; Overpeck et al., 1992; Becerra et al., 1993; Herman et al., 1997). No Brasil, essas iniciativas são recentes (Almeida, 1994; Aerts et al., 1995; Guerra et al., 1995; Accioly, 1997), tendo sido viabilizadas com a implantação, pelo Ministério da Saúde, do Sistema de Informações sobre Nascidos Vivos (SINASC) em 1990 (Mello-Jorge et al., 1996).

O procedimento de linkage entre nascimentos e óbitos infantis aumenta a potencialidade de análise epidemiológica das informações desses sistemas, tornando viável: 1) o estudo da mortalidade infantil em coortes de nascidos vivos de base populacional; 2) a estimativa direta da probabilidade de morte infantil antes de completar um ano e nos períodos neonatal e pós-neonatal; 3) a estimativa da proba- bilidade de morte em subgrupos de recémnascidos, categorizados segundo as variáveis presentes na Declaração de Nascido Vivo (DN); 4) o estudo da existência de associação e da magnitude desta associação entre as variáveis independentes presentes na DN e a mortalidade neonatal e pós-neonatal; 5) o uso de fontes de dados secundárias, disponíveis nos sistemas de informações de mortalidade e nascidos vivos para realização de estudos analíticos longitudinais (Almeida, 1994; Herman et al., 1997).

O objetivo deste artigo foi investigar fatores de risco para os componentes neonatal e pósneonatal da mortalidade infantil no município de Goiânia, utilizando como potenciais fatores de risco as variáveis presentes na DN.

\section{Material e métodos}

\section{Área e população de estudo}

Este estudo foi realizado no Município de Goiânia, Goiás - 922.222 habitantes no censo de 1991 (IBGE, 1991) - localizado na Região Centro-Oeste do Brasil. A população de estudo foi compreendida por todos os nascimentos vivos com DN preenchida, ocorridos entre 01/01/ 1992 e $31 / 12 / 1992$

\section{- Nascidos vivos}

A fonte de dados dos nascidos vivos foi a DN documento básico do SINASC, utilizado no Município de Goiânia desde 1990 (Mello-Jorge et al., 1993). Para a definição da coorte de nascidos vivos no ano de 1992, foram considerados, como critérios de inclusão no estudo, os nascidos vivos cujo local de nascimento e endereço de residência da mãe foram o Município de Goiânia. O total de nascidos vivos que tiveram uma DN preenchida foi de 20.981. Este valor representa uma cobertura aproximada do SINASC de Goiânia da ordem de $87 \%$, de acordo com o número esperado de nascidos vivos para o município no ano de 1992 (Mello-Jorge et al., 1996).

\section{- Óbitos}

A fonte de dados utilizada foi a declaração de óbito (DO). Foram considerados, no estudo, os óbitos infantis registrados nos arquivos de 1992 e 1993 que satisfaziam os seguintes critérios: 1) nascimento ocorrido em 1992, 2) local de nascimento em Goiânia e 3) endereço de residência no Município de Goiânia. Foram excluídos do estudo: 1) recém-nascidos que 
morreram em 1992, mas nasceram em 1991, por pertencerem a outra coorte de nascimento; 2) óbitos infantis de residentes em Goiânia, mas cujo município de nascimento não foi esta cidade, e 3) óbitos infantis de não residentes em Goiânia, apesar de terem sido codificados como de residentes no município pela equipe do Sistema de Informação sobre Mortalidade (SIM). Ao final, foram incluídos no estudo e submetidos ao procedimento de linkage um total de 349 óbitos infantis - 297 ocorridos em 1992 e 52 no ano de 1993.

\section{- Linkage entre bancos de dados}

Cada DO foi emparelhada com a respectiva $\mathrm{DN}$, de acordo com os seguintes procedimentos: 1) uma pesquisa automática no banco de dados de nascidos vivos, utilizando campos comuns à DN e à DO (data de nascimento, sexo e bairro de residência); 2) pesquisa manual nos arquivos de DN visando confirmar a(s) DN(s) identificada(s) no procedimento automático, utilizando a variável nome da mãe; 3) pesquisa em cartórios de registro civil e hospitais, no caso de insucesso nas etapas anteriores.

A partir do procedimento de linkage, foi definida a condição de sobrevivência da coorte retrospectiva de nascidos vivos em Goiânia no ano de 1992, identificando-se os nascidos vivos que evoluíram para óbito infantil nos períodos neonatal e pós-neonatal e os sobreviventes. O procedimento de linkage adotado não conseguiu identificar a DN de sete óbitos infantis $-2,0 \%$ do total. Para a análise dos dados, foi considerado um total de 20.981 nascidos vivos, tendo 342 destes evoluído para o óbito antes de completar o primeiro ano de vida.

\section{- Variáveis analisadas}

a) Variável dependente: probabilidade de morte nos períodos neonatal e pós-neonatal. b) Variáveis de exposição: grau de instrução da mãe (superior, ensino fundamental ou ensino médio, ensino fundamental incompleto e sem instrução), categoria do hospital de nascimento da criança (privado, privado e filantrópico conveniado ao Sistema Único de Saúde-SUS e público-estatal), idade materna (menor que 20 anos, 20 a 34 anos e igual ou superior a 35 anos), peso ao nascer (menor que 2.500 gramas e igual ou superior a 2.500 gramas), duração da gestação (menor que 37 semanas, 37 a 42 semanas e maior que 42 semanas), gênero (masculino e feminino), tipo de gravidez (única e múltipla) e tipo de parto (operatório e espontâneo).

\section{- Processamento dos dados}

Para o processamento eletrônico e análise, os arquivos de dados foram transferidos para os aplicativos de análise estatística EPIINFO 6.03 (Dean et al., 1996) e SAS (SAS Institute Inc., 1985). Dois bancos de dados primários foram construídos, bem como um terceiro, resultante do procedimento de linkage: arquivo de nascidos vivos, arquivo de óbitos e arquivo de dados vinculados.

\section{- Análise de dados}

a) Análise bivariada: foram realizadas distribuição de freqüência, tabulação cruzada entre variáveis, estimativa da probabilidade de óbito no período neonatal e pós-neonatal, segundo as categorias de exposição, odds ratio (OR) e respectivos intervalos de confiança de 95\% (IC 95\%), cálculo da significância estatística da associação, utilizando o teste de Qui-Quadrado. b) Análise multivariada: o procedimento estatístico para o ajustamento dos potenciais efeitos de confusão foi a regressão logística. Foram construídos modelos hierarquizados para cada um dos componentes da mortalidade infantil (Fuchs et al., 1996). Nesta estratégia de análise, consideraram-se, para a ordenação da introdução das variáveis no modelo, o nível hierárquico de cada variável de exposição na cadeia de determinação da mortalidade infantil. A hierarquização das variáveis obedeceu à seguinte seqüência: 1) variáveis situadas no nível hierárquico de maior poder de determinação da mortalidade infantil - grau de instrução da mãe e tipo de hospital; 2) variáveis situadas no nível hierárquico intermediário - tipo de parto e idade da mãe; 3) variáveis situadas no nível hierárquico mais próximo do efeito - peso ao nascer, duração da gestação e tipo de gravidez. A variável gênero do recém-nascido foi introduzida no modelo como variável de controle. Para a seleção do modelo final, foram incluídas no modelo todas as variáveis com $\mathrm{p}<0,25$ na análise bivariada, além das variáveis de importância clínico-epidemiológica descritas na literatura revisada (Hosmer \& Lemeshow, 1989).

\section{Resultados}

Do total de 342 óbitos infantis, 231 (67,5\%) ocorreram no período neonatal - antes de os recém-nascidos completarem 28 dias de vida - e 111 (32,5\%), no período pós-neonatal - de 28 dias a um ano incompleto. A probabilidade de morte antes de completar um ano de vida foi 
de 16,3 óbitos por mil nascidos vivos, sendo 11,0 por mil, no período neonatal, e 5,3 por mil, no período pós-neonatal. A taxa de mortalidade infantil no Município de Goiânia para o ano de 1992 foi 19,9 (436/21.963) óbitos por mil nascidos vivos. As diferenças entre a probabilidade de morte e a taxa de mortalidade infantil deveram-se às exclusões no numerador e denominador descritas anteriormente.

$\mathrm{Na}$ análise bivariada, foram identificadas associações estatisticamente significativas entre mortalidade no período neonatal para os seguintes subgrupos de nascidos vivos: nascidos vivos em hospital público-estatal apresentaram risco de morte mais elevado que os nascidos em hospital privado não conveniados ao SUS (OR = 2,28; IC 95\% 1,57-3,32); nascidos vivos com baixo peso ao nascer (menor que $2.500 \mathrm{~g}$ ) apresentaram risco de morte elevado quando comparados aos nascidos com peso igual ou superior a $2.500 \mathrm{~g}(\mathrm{OR}=27,60$; IC $95 \%$ 20,40-37,33); nascidos vivos prematuros (duração da gestação inferior a 37 semanas) apresentaram um risco elevado de morte em relação aos nascidos de termo (OR = 37,68; IC 95\% 28,35-50,06); nascidos vivos de gravidez múltipla apresentaram um risco de morte 3,6 vezes superior quando comparados aos nascidos vivos de gravidez única e aos nascidos vivos por parto cesariana apresentaram um risco de morte $50 \%$ inferior aos nascidos vivos por parto espontâneo (OR = 0,48; IC 95\% 0,37-0,63). As variáveis idade da mãe, gênero do recém-nascido e grau de instrução da mãe não estiveram associadas a um maior risco de mortalidade no período neonatal.

A análise bivariada mostrou associação estatisticamente significativa entre a mortalidade no período pós-neonatal e os seguintes subgrupos de nascidos vivos: nascidos vivos em hospital público-estatal (OR = 4,59; IC 95\% 2,12$9,95)$ e em hospital privado conveniado ao SUS $(\mathrm{OR}=3,67$; IC 95\% 1,76-7,64) apresentaram risco de morte superior aos nascidos em hospital privado não conveniado ao SUS; nascidos vivos de mãe sem instrução (OR = 11,10; IC 95\% 2,39$51,49)$ e de mãe com primeiro grau incompleto $(\mathrm{OR}=5,36$; IC 95\% 1,31-21,91) apresentaram risco de morte elevado em relação aos nascidos vivos de mãe com nível superior de escolaridade; nascidos vivos de baixo peso ao nascer apresentaram um maior risco de morte quando comparados aos nascidos com peso adequado ao nascer $(\mathrm{OR}=5,52$; IC 95\% 3,57-8,54); nascidos vivos prematuros apresentaram risco de morte superior ao dos nascidos vivos de termo (OR = 4,98; IC 95\% 2,95-8,41); nascidos vivos de gravidez múltipla apresentaram risco de morte superior em relação aos nascidos vivos de gravidez única (OR = 2,96; IC 95\% 1,29-6,79); e nascidos vivos por parto cesariana apresentaram um risco de morte $45 \%$ inferior ao dos nascidos por parto espontâneo $(\mathrm{OR}=0,55$; $\mathrm{IC}$ $95 \%$ 0,37-0,80). As variáveis gênero do recémnascido e idade da mãe não mostraram associação estatisticamente significativa com a mortalidade no período pós-neonatal.

No período neonatal, a análise multivariada mostrou os seguintes resultados: 1) nascidos vivos em hospital público-estatal apresentaram um risco de morte no período neonatal 2,3 vezes superior ao dos nascidos em hospital privado não conveniado ao SUS; 2) nascidos vivos por parto cesariana tiveram um risco de morte neonatal $42 \%$ inferior ao dos nascidos por parto espontâneo; 3 ) nascidos vivos prematuros tiveram um risco de morte no período neonatal 8,9 vezes maior do que os de termo; 4) nascidos vivos com baixo peso ao nascer apresentaram um risco 8,9 vezes maior de morte neonatal quando comparados aos de peso igual ou superior a $2.500 \mathrm{~g}$ (Tabela 1 ). As variáveis gênero do recém-nascido e idade da mãe não apresentaram significância estatística e não atuaram como fatores de confusão para as demais variáveis. A variável tipo de gravidez, apesar de não apresentar significância estatística, permaneceu no modelo como variável controle.

O modelo da análise multivariada para o período pós-neonatal mostrou os seguintes resultados: 1) nascidos vivos de mães sem instrução apresentaram risco de morte 6,3 vezes superior ao dos nascidos de mãe com nível superior; 2) nascidos vivos em hospital público-estatal apresentaram um risco de morte 2,7 vezes superior em relação aos nascidos em hospital privado não conveniado ao SUS; 3) nascidos vivos com baixo peso ao nascer apresentaram um risco de mortalidade 3,1 vezes superior ao dos nascidos vivos com peso adequado ao nascer (Tabela 2). As variáveis gênero do recémnascido e idade da mãe não apresentaram associação estatisticamente significativa e não atuaram como fatores de confusão para as demais variáveis, o que justificou a exclusão do modelo. As variáveis duração da gestação e tipo de gravidez permaneceram no modelo como variáveis de controle, apesar de não apresentarem significância estatística.

\section{Discussão}

A comparação dos resultados observados neste estudo com os de outros trabalhos que utili- 
Fatores de risco para mortalidade no período neonatal, ajustados por regressão logística.

\begin{tabular}{|c|c|c|c|c|}
\hline Variável & $\begin{array}{l}\text { Odds Ratio } \\
\text { (não ajustado) }\end{array}$ & $\begin{array}{l}\text { Intervalo de } \\
\text { confiança }(95 \%)\end{array}$ & $\begin{array}{l}\text { Odds Ratio } \\
\text { (ajustado) }\end{array}$ & $\begin{array}{l}\text { Intervalo de } \\
\text { confiança }(95 \%)\end{array}$ \\
\hline \multicolumn{5}{|l|}{ Tipo de hospital } \\
\hline Privado não SUS & 1,00 & - & 1,00 & - \\
\hline Público-Estatal & $2,28^{\star \star}$ & $1,57-3,32$ & $2,28^{\star \star}$ & $1,57-3,32$ \\
\hline Privado conveniado SUS & 1,06 & $0,74-1,53$ & 1,06 & $0,74-1,53$ \\
\hline \multicolumn{5}{|l|}{ Tipo de parto } \\
\hline Espontâneo & 1,00 & - & 1,00 & - \\
\hline Cesariana & $0,48^{\star *}$ & $0,37-0,63$ & $0,58^{\star *}$ & $0,43-0,78$ \\
\hline \multicolumn{5}{|c|}{ Duração da gestação (semanas) } \\
\hline $37-41$ & 1,00 & - & 1,00 & - \\
\hline$<37$ & $37,68^{\star *}$ & $28,35-50,06$ & $8,94 * \star$ & $5,85-13,67$ \\
\hline$\geq 42$ & 1,64 & $0,52-5,20$ & 2,35 & $0,73-7,55$ \\
\hline \multicolumn{5}{|l|}{ Peso ao nascer (gramas) } \\
\hline$\geq 2.500$ & 1,00 & - & 1,00 & - \\
\hline$<2.500$ & $27,60^{* *}$ & $20,40-37,33$ & $8,92^{\star \star}$ & $5,77-13,79$ \\
\hline \multicolumn{5}{|l|}{ Tipo de gravidez } \\
\hline Única & 1,00 & - & 1,00 & - \\
\hline Múltipla & $3,56^{\star *}$ & $2,09-6,07$ & 0,62 & $0,32-1,20$ \\
\hline
\end{tabular}

* $p<0,05$

$* * p<0,01$

Tabela 2

Fatores de risco para mortalidade no período pós-neonatal, ajustados por regressão logística.

\begin{tabular}{|c|c|c|c|c|}
\hline Variável & $\begin{array}{l}\text { Odds Ratio } \\
\text { (não ajustado) }\end{array}$ & $\begin{array}{l}\text { Intervalo de } \\
\text { confiança }(95 \%)\end{array}$ & $\begin{array}{l}\text { Odds Ratio } \\
\text { (ajustado) }\end{array}$ & $\begin{array}{l}\text { Intervalo de } \\
\text { confiança (95\%) }\end{array}$ \\
\hline \multicolumn{5}{|l|}{ Instrução da mãe } \\
\hline Superior & 1,00 & - & 1,00 & - \\
\hline Fundamental ou médio & 2,32 & $0,55-9,82$ & 1,65 & $0,38-7,22$ \\
\hline Fundamental incompleto & $5,36^{\star}$ & $1,31-21,91$ & 2,97 & $0,67-13,16$ \\
\hline Sem-instrução & $11,10^{*}$ & $2,39-51,49$ & $6,25^{\star}$ & $1,25-31,27$ \\
\hline \multicolumn{5}{|l|}{ Tipo de hospital } \\
\hline Privado não SUS & 1,00 & - & 1,00 & - \\
\hline Público-Estatal & $4,59 * *$ & $2,12-9,95$ & $2,65^{\star}$ & $1,13-6,23$ \\
\hline Privado conveniado SUS & $3,67^{\star *}$ & $1,76-7,64$ & 2,14 & $0,96-4,77$ \\
\hline \multicolumn{5}{|l|}{ Peso ao nascer (gramas) } \\
\hline$\geq 2.500$ & 1,00 & - & 1,00 & - \\
\hline$<2.500$ & $5,52^{\star \star}$ & $3,57-8,54$ & $3,12^{\star \star}$ & $1,67-5,84$ \\
\hline \multicolumn{5}{|c|}{ Duração da gestação (semanas) } \\
\hline $37-41$ & 1,00 & - & 1,00 & - \\
\hline$<37$ & $4,98^{\star *}$ & $2,95-8,41$ & 2,03 & $0,97-4,26$ \\
\hline \multicolumn{5}{|l|}{ Tipo de gravidez } \\
\hline Única & 1,00 & - & 1,00 & - \\
\hline Múltipla & $2,96^{\star *}$ & $1,29-6,79$ & 1,21 & $0,42-3,49$ \\
\hline
\end{tabular}

* $p<0,05$

$\star * p<0,01$ 
zam a mesma abordagem metodológica ressalta os seguintes aspectos: com relação à categoria do hospital de nascimento, os nascidos vivos em hospital público-estatal - como observado nos artigos revisados que investigaram esta variável - apresentam um maior risco de morte nos períodos neonatal e pós-neonatal (Becerra et al., 1993; Almeida, 1994). A categoria do hospital de nascimento da criança pode ser entendida como um indicador de duas dimensões: primeiro, como um marcador sócioeconômico da família dos nascidos vivos, refletindo o perfil de consumo de bens e serviços, as características culturais e nutricionais e o acesso a serviços de saúde, incluindo a assistência pré-natal desse subgrupo populacional; em segundo lugar, a categoria do hospital pode ser tomada como um indicador da qualidade da assistência prestada durante a gestação, no momento do parto e ao recém-nascido (Becerra et al., 1993).

Quanto ao grau de instrução da mãe, os resultados obtidos são coerentes com os de outros autores (Ferreira, 1990; Macharelli \& Oliveira, 1991; Almeida, 1994; Arntzen et al., 1996; Din-Dzietham \& Hertz-Picciotto, 1997). Assim, o grau de instrução é considerado por diversos autores como um marcador da condição sócio-econômica da mãe e de sua família. Além desse enfoque, o nível educacional da mãe pode ser compreendido também como um fator relacionado ao perfil cultural e a comportamentos ligados aos cuidados de saúde que têm um efeito importante na determinação da mortalidade infantil (Ferreira, 1990; Adetunji, 1995).

O baixo peso ao nascer e a prematuridade são os fatores de risco descritos na literatura que apresentam a maior força de associação com a mortalidade infantil. O peso ao nascer e a duração da gestação não devem, no entanto, ser estudados como fatores de risco isolados, mas como mediadores através dos quais atuam diversos determinantes e condicionantes da mortalidade infantil, tais como: escolaridade e características sócio-econômicas da mãe, morbidade materna, características biológicas e hábitos de vida da mãe, acesso a serviços de saúde durante a gestação e qualidade desses serviços, entre outros. Estas variáveis devem ser consideradas marcadores de saúde capazes de predizer os riscos de mortalidade nos períodos neonatal e pós-neonatal de uma determinada criança. Recém-nascidos com baixo peso são mais vulneráveis a problemas como imaturidade pulmonar e transtornos metabólicos, que podem causar ou agravar alguns eventos que acometem os recém-nascidos, aumentan- do o risco de mortalidade (Buchalla, 1988). Os principais mecanismos relacionados à etiologia da prematuridade - duração da gestação inferior a 37 semanas - são a ruptura prematura das membranas, o trabalho de parto prematuro, interrupção precoce da gestação em razão de problemas relacionados à mãe ou ao feto, associados a outros fatores, tais como: tabagismo, sangramento vaginal e doença hipertensiva específica da gestação. A prematuridade tem sido referida como um dos fatores mais importantes para a mortalidade perinatal e neonatal precoce (Berkowitz \& Papiernik, 1993).

Em concordância com o observado por outros autores, na coorte de nascidos vivos em Goiânia, a magnitude da associação do baixo peso ao nascer é mais acentuada na mortalidade neonatal, embora persista uma associação de menor magnitude com a mortalidade pósneonatal. A prematuridade, quando ajustada por outras variáveis do modelo multivariado, persiste enquanto um fator de risco importante no período neonatal e perde a significância estatística no período pós-neonatal (Semenciw et al., 1986).

Os nascidos vivos de gravidez múltipla apresentam uma alta incidência de baixo peso ao nascer e prematuridade. Por essa razão, em grande parte dos estudos de fatores de risco para mortalidade infantil, os gemelares são excluídos da análise. No presente trabalho, optou-se pela permanência deste subgrupo de nascidos vivos, controlando-se o seu efeito na análise multivariada. A perda da significância estatística da variável tipo de gravidez na análise multivariada deveu-se a sua introdução no modelo no mesmo nível hierárquico que as variáveis peso ao nascer e duração da gestação. Estas, em virtude da grande magnitude dos seus riscos, esvaziam a associação do tipo de gravidez com a mortalidade infantil nos períodos neonatal e pós-neonatal.

Na coorte de nascidos vivos em Goiânia, o parto cesariana mostrou-se como um fator de proteção para a mortalidade no período neonatal, mesmo controlando o efeito de variáveis de confusão, como o peso ao nascer e a categoria do hospital de nascimento da criança (Tabela 1). Esse resultado, no entanto, não parece lógico e não é consensual na literatura revisada sobre o efeito protetor do parto operatório para a mortalidade neonatal. Na análise desta associação - parto cesariana e mortalidade neonatal - estratificada pelo peso ao nascer e tipo de hospital, observou-se que o efeito protetor da cesariana concentra-se em dois subgrupos de nascidos vivos: baixo peso ao nascer $(<2.500 \mathrm{~g})$ e nascidos vivos em hospital privado não con- 
veniado ao SUS (Tabelas 3 e 4). Embora não haja um consenso na literatura sobre a indicação do parto cesariana para recém-nascidos de muito baixo peso, alguns autores observaram uma redução da mortalidade neonatal nestes grupos de recém-nascidos submetidos ao parto cesariana, quando comparados aos nascidos por parto espontâneo (Bottoms et al., 1997; Jonas \& Lumley, 1997). Com relação aos nascidos vivos em hospital privado, o efeito protetor do parto cesariana pode estar relacionado a outros fatores, tais como: nível sócio-econômico da mãe, acesso a um serviço pré-natal e de atenção ao parto e ao recém-nascido de melhor qualidade. Estudos realizados no Brasil mostram uma correlação positiva entre as taxas de cesariana e o nível sócio-econômico das mães (Faúndes \& Cecatti, 1991). Como o presente estudo não foi delineado para investigar especificamente o papel da variável tipo de parto na causalidade da mortalidade neonatal, estudos adicionais são necessários para abordar esta questão.

A análise de fatores de risco para mortalidade infantil, como mostram os resultados deste artigo, deve levar em conta a distinção entre

Tabela 3

Tipo de parto estratificado pelo peso ao nascer, mortalidade no período neonatal.

\begin{tabular}{|c|c|c|c|c|}
\hline Variável & Óbitos & Sobreviventes & Odds Ratio & $\begin{array}{l}\text { Intervalo de } \\
\text { confiança }(95 \%)\end{array}$ \\
\hline \multicolumn{5}{|c|}{ Peso ao nascer $<2.500 \mathrm{~g}$} \\
\hline \multicolumn{5}{|c|}{ Tipo de parto } \\
\hline Espontâneo & 87 & 678 & 1,00 & - \\
\hline Cesariana & 33 & 563 & $0,46^{\star \star}$ & $0,29-0,71$ \\
\hline \multicolumn{5}{|c|}{ Peso ao nascer $\geq 2.500 \mathrm{~g}$} \\
\hline \multicolumn{5}{|c|}{ Tipo de parto } \\
\hline Espontâneo & 27 & 8256 & 1,00 & - \\
\hline Cesariana & 37 & 10794 & 1,05 & $0,62-1,78$ \\
\hline
\end{tabular}

** $\mathrm{p}<0,01$

Qui-Quadrado para avaliação de heterogeneidade entre os estratos $=7,87 ; p=0,01$.

Tabela 4

Tipo de parto estratificado pelo tipo de hospital, mortalidade no período neonatal.

\begin{tabular}{|c|c|c|c|c|}
\hline Variável & Óbitos & Sobreviventes & Odds Ratio & $\begin{array}{l}\text { Intervalo de } \\
\text { confiança (95\%) }\end{array}$ \\
\hline \multicolumn{5}{|c|}{ Hospital público-estatal } \\
\hline \multicolumn{5}{|l|}{ Tipo de parto } \\
\hline Espontâneo & 60 & 3156 & 1,00 & - \\
\hline Cesariana & 21 & 1136 & 0,97 & $0,57-1,65$ \\
\hline \multicolumn{5}{|c|}{ Hospital privado SUS } \\
\hline \multicolumn{5}{|l|}{ Tipo de parto } \\
\hline Espontâneo & 59 & 4806 & 1,00 & - \\
\hline Cesariana & 39 & 6307 & $0,50 * \star$ & $0,33-0,77$ \\
\hline \multicolumn{5}{|c|}{ Hospital privado } \\
\hline \multicolumn{5}{|l|}{ Tipo de parto } \\
\hline Espontâneo & 15 & 887 & 1,00 & - \\
\hline Cesariana & 25 & 3929 & $0,38^{\star *}$ & $0,19-0,76$ \\
\hline
\end{tabular}

** $\mathrm{p}<0,01$

Qui-Quadrado para avaliação de heterogeneidade entre os estratos $=6,27 ; p=0,04$. 
os componentes neonatal e pós-neonatal, a fim de ressaltar as diferenças nos fatores e na magnitude da associação destes com a mortalidade infantil. As causas de morte e o perfil dos fatores de risco apresentam diferenças entre os dois períodos, especialmente com relação às variáveis sócio-econômico-culturais, como a escolaridade da mãe.

\section{Conclusão}

A realização deste estudo e de outros que utilizaram o procedimento de linkage de bancos de dados de nascidos vivos e óbitos infantis mostra a viabilidade e as vantagens dessa estratégia, ainda pouco explorada em nosso meio, tornando possível a realização de estudos analíticos longitudinais, utilizando-se como fonte de dados os sistemas de informação em saúde disponíveis no Brasil.

Finalmente, essa estratégia de análise possibilita o monitoramento da prevalência dos fatores de risco para mortalidade infantil e das mudanças na magnitude desses fatores nas coortes de nascidos vivos nos anos posteriores a 1992. Este estudo mostrou também que alguns subgrupos de nascidos vivos - prematuros, baixo peso ao nascer, nascidos em hospital público-estatal e filhos de mãe sem instrução formal -, que constituem grupos de risco para mortalidade infantil nos período neonatal ou pós-neonatal, devem ser priorizados pelos serviços de saúde, por meio de ações e intervenções que reduzam o impacto desses fatores na qualidade de vida das crianças, evitando intercorrências, seqüelas e morte no primeiro ano de vida.

\section{Agradecimentos}

Agradecemos ao Dr. Gélcio Sisteroli de Carvalho, do Departamento de Morbi-Mortalidade da Secretaria de Saúde do Estado de Goiás, por ter tornado os dados disponíveis; à Professora Celina Maria Turchi Martelli, pelas críticas e sugestões durante a análise dos dados, e aos assistentes de pesquisa Aline Cristina de Andrade e João B. Peres Júnior, pela valiosa contribuição nas fases de coleta, conferência e digitação dos dados. Agradecemos também à CAPES, pela bolsa de estudos que propiciou a conclusão deste trabalho.

\section{Referências}

ACCIOLY, M. C., 1997. Determinantes da mortalidade neonatal em Belo Horizonte (1993): Subsídios para o planejamento da atenção materno-infantil. In: 5o Congresso Brasileiro de Saúde Coletiva e 5oㅡ Congresso Paulista de Saúde Pública, Resumos, p. 307. Águas de Lindóia: ABRASCO.

ADETUNJI, J. A., 1995. Infant mortality and mother's education in Ondo State, Nigeria. Social Science \& Medicine, 40:253-263.

AERTS, D.; LIMA, M. \& HIRAKATA, V., 1995. Perfil da mortalidade neonatal em Porto Alegre: Um estudo integrado do SIM-SINASC. In: 3o Congresso Brasileiro de Epidemiologia, 2o Congresso IberoAmericano de Epidemiologia, 1o Congresso Latino-Americano de Epidemiologia, Resumos, p. 296. Salvador: ABRASCO.

ALMEIDA, M. F., 1994. Mortalidade Neonatal em Santo André. Tese de Doutorado, São Paulo: Faculdade de Saúde Pública, Universidade de São Paulo.

ARNTZEN, A.; MOUN, T.; MAGNUS, P. \& BAKKETEIG, L. S., 1996. The association between maternal education and post-neonatal mortality. Trends in Norway, 1968-1991. International Journal of Epidemiology, 25:578-584.

BATTELLINO, V. L. J. \& BENNUN, F. R., 1991. Niveles, tendencias y estructura de la mortalidad infantil en la Provincia de Córdoba (Argentina). Cuadernos Médico Sociales, 56:45-58.

BECERRA, J. E.; ATRASH, H. K.; PÉREZ, N. \& SALICETI, J. A., 1993. Low birth weight and infant mortality in Puerto Rico. American Journal of Public Health, 83:1572-1576 
BERKOWITZ, G. S. \& PAPIERNIK, E., 1993. Epidemiology of pre-term birth. Epidemiologic Review, 15:414-443.

BOTTOMS, S. F.; PAUL, R. H.; IAMS, J. D.; MERCER, B. M.; THOM, E. A.; ROBERTS, J. M.; CARITIS, S. N.; MOAWAD, A. H.; VAN DORSTEN, J. P.; HAUTH, J. C.; THURNAU, G. R.; MIODOVINIK, M.; MEIS, P. M. \& McNELLIS, D., 1997. Obstetric determinants of neonatal survival: Influence of willingness to perform cesarean delivery on survival of extremely low-birth-weight infants. American Journal of Obstetetrics and Gynecology, 176:960-966.

BUCHALLA, C. M., 1988. Estudo de um Grupo de Recém-Nascidos em Maternidades: Suas Características e a Mortalidade do Período Neonatal Preco$c e$. Dissertação de Mestrado, São Paulo: Faculdade de Saúde Pública, Universidade de São Paulo.

CASTELLANOS, P. L., 1991. Sistemas Nacionales de Vigilancia de la Situación de la Salud Según Condiciones de Vida y del Impacto de las Acciones de Salud y Bienestar. Washington, D.C.: Organización Panamericana de la Salud. (mimeo.)

CÉSAR, C. L. G., 1990. Fatores de risco associados à mortalidade infantil em duas áreas da região metropolitana de São Paulo (Brasil), 1984-1985: Proposta de instrumentos preditivos. Revista de Saúde Pública, 24:300-310.

DEAN, A. G.; DEAN, J. A.; BURTON, A. H. \& DICKER, R. C., 1996. EPIINFO, Versão 6.03: A Word Processor, Database and Statistics Program for Epidemiology on Microcomputers. Atlanta: Centers for Disease Control and Prevention.

DIN-DZIETHAM, R. \& HERTZ-PICCIOTTO, I., 1997. Relationship of education to the racial gap in neonatal and post-neonatal mortality. Archives of Pediatrics and Adolescent Medicine, 151:787-792.

FAÚNDES, A. \& CECATTI, J. G., 1991. A operação cesárea no Brasil. Incidência, tendências, causas, conseqüências e propostas de ação. Cadernos de Saúde Pública, 7:150-173.

FERREIRA, C. E. C., 1990. Mortalidade Infantil e Desigualdade Social em São Paulo. Tese de Doutorado, São Paulo: Faculdade de Saúde Pública, Universidade de São Paulo.

FUCHS, S. C.; VICTORA, C. G. \& FACHEL, J., 1996. Modelo hierarquizado: Uma proposta de modelagem aplicada à investigação de fatores de risco para diarréia grave. Revista de Saúde Pública, 30:168178.

GUERRA, H. L.; MENEZES, F. R. \& CORREA, P. R. L., 1995. Mortalidade neonatal e pós-neonatal na área de risco do Distrito Sanitário Noroeste de Belo Horizonte. In: 3o Congresso Brasileiro de Epidemiologia, 2o Congresso Ibero-Americano de Epidemiologia, 1 Congresso Latino-Americano de Epidemiologia, Resumos, p. 5. Salvador: ABRASCO.

HERMAN, A. A.; McCARTHY, B. J.; BAKEWELL, J. M.; WARD, R. H.; MUELLER, B. A.; MACONOCHIE, N. E.; READ, A. W.; ZADKA, P. \& SKAJAERVEN, R., 1997. Data linkage methods used in maternally- linked birth and infant death surveillance data sets from the United States (Georgia, Missouri, Utah and Washington State), Israel, Norway, Scotland and Western Australia. Paediatric and Perinatal Epidemiology, 11(Sup. 1):5-22.

HOSMER, D. W. \& LEMESHOW, S., 1989. Applied Logistic Regression. New York: John Wiley \& Sons.

JONAS, H. A. \& LUMLEY, J. M., 1997. The effect of mode of delivery on neonatal mortality in very low birthweight infants born in Victoria, Australia: Caesarian section is associated with increased survival in breech-presenting, but not vertex-presenting, infants. Paediatric and Perinatal Epidemiology, 11:181-199.

MACHARELLI, C. A. \& OLIVEIRA, L. R., 1991. Perfil do risco de óbito de crianças menores de um ano residentes em localidade do Estado de São Paulo, 1987. Revista de Saúde Pública, 25:121-128.

MARANHÃO, A. G. K.; JOAQUIM, M. M. C.; SIU, C.; KALUME, P.; CASTILLO, O. \& LEAL, M. C., 1999. Mortalidade perinatal e neonatal no Brasil. TEMA. Radis, 17:6-17.

MELLO-JORGE, M. H. P.; GOTLIEB, S. L. D. \& OLIVEIRA, H., 1996. O sistema de informação sobre nascidos vivos: Primeira avaliação dos dados brasileiros. Informe Epidemiológico do SUS, 2:15-48.

MELLO-JORGE, M. H. P.; GOTLIEB, S. L. D.; SOBOLL, M. L. M. S.; ALMEIDA, M. F. \& LATORRE, M. R. D. O., 1993. Avaliação do sistema de informação sobre nascidos vivos e o uso de seus dados em epidemiologia e estatísticas de saúde. Revista de Saúde Pública, 27(Sup. 6):1-44.

MONTEIRO, C. A. \& NAZÁRIO, C. L., 1995. Declínio da mortalidade infantil e eqüidade social: O caso da cidade de São Paulo entre 1973 e 1993. In: Velhos e Novos Males da Saúde no Brasil. A Evolução do País e de suas Doenças (C. A. Monteiro, org.), pp. 173-185, São Paulo: Editora Hucitec/NUPENS/Universidade de São Paulo.

OVERPECK, M. D.; HOFFMAN, H. J. \& PRAGER, K., 1992. The lowest birth-weight infants and the US infant mortality rate: NCHS 1983 linked birth/infant death data. American Journal of Public Health, 82:441-444.

PAIM, J. S. \& COSTA, M. C. N., 1993. Decréscimo e desigualdade da mortalidade infantil: Salvador, 19801988. Boletín de la Oficina Sanitaria Panamericana, 114:415-428.

SAS INSTITUTE INC., 1985. SAS - Introductory Guide for Personal Computers. Version 6. Cary: SAS Institute Inc.

SEMENCIW, R. M.; MORRISON, H. I.; LINDSAY, J.; SILINS, J.; SHERMAN, G. J.; MAO, Y. \& WIGLE, D. T., 1986. Risk factors for post-neonatal mortality: Results from a record linkage study. International Journal of Epidemiology, 15:369-372.

SIMÕES, C. C. S. \& MONTEIRO, C. A., 1995. Tendência secular e diferenciais regionais da mortalidade infantil no Brasil. In: Velhos e Novos Males da Saúde no Brasil. A Evolução do País e de suas Doenças (C. A. Monteiro, org.), pp. 153-156, São Paulo: Editora Hucitec/ NUPENS/Univesidade de São Paulo. 